\title{
Upper Airway Dilator Muscle Weakness Following Intermittent and Sustained Hypoxia in the Rat: Effects of a Superoxide Scavenger
}

\author{
J. R. SKELLY ${ }^{1}$, S. C. ROWAN ${ }^{1}$, J. F. X. JONES ${ }^{1}$, K. D. O'HALLORAN ${ }^{1}$ \\ ${ }^{1}$ UCD School of Medicine and Medical Science, Health Sciences Center, University College \\ Dublin, Dublin, Ireland
}

Received June 8, 2012

Accepted November 2, 2012

On-line December 13, 2012

\begin{abstract}
Summary
Obstructive sleep apnoea syndrome (OSAS) is a common disorder associated with upper airway muscle dysfunction. Agents that improve respiratory muscle performance may have considerable therapeutic value. We examined the effects of acute exposure to sustained and intermittent hypoxia on rat pharyngeal dilator muscle function. Additionally, we sought to test the efficacy of antioxidant treatment in ameliorating or preventing hypoxia-related muscle dysfunction. Isometric contractile and endurance properties of isolated rat sternohyoid muscle bundles were examined at $35{ }^{\circ} \mathrm{C}$ in vitro. Muscle bundles were exposed to one of four gas treatments: hyperoxia (control), sustained hypoxia (SH), intermittent hypoxia (IH) or hypoxia/reoxygenation (HR), in the absence or presence of the superoxide scavenger - Tempol (10 mM). Stress-frequency relationship was determined in response to electrical stimulation $(10-100 \mathrm{~Hz}$ in increments of $10-20 \mathrm{~Hz}$, train duration: $300 \mathrm{~ms}$ ). Muscle performance was also assessed during repetitive muscle stimulation ( $40 \mathrm{~Hz}, 300 \mathrm{~ms}$ every $2 \mathrm{~s}$ for $2.5 \mathrm{~min}$ ). Compared to control, IH and HR treatments significantly decreased sternohyoid muscle force. The negative inotropic effect of the two gas protocols was similar, but both were of lesser magnitude than the effects of $\mathrm{SH}$. SH, but not $\mathrm{IH}$ and HR, increased muscle fatigue. Tempol significantly increased sensitivity to stimulation in all muscle preparations and caused a leftward shift in the stressfrequency relationship of $\mathrm{IH}$ and $\mathrm{SH}$ treated muscles. Tempol did not ameliorate sternohyoid muscle fatigue during $\mathrm{SH}$. We conclude that Tempol increases upper airway muscle sensitivity to stimulation but only modestly ameliorates respiratory muscle weakness during intermittent and sustained hypoxic conditions in vitro. Respiratory muscle fatigue during sustained hypoxia appears unrelated to oxidative stress.
\end{abstract}

\section{Key words}

Antioxidants • Fatigue • Hypoxia/re-oxygenation • Intermittent hypoxia - Superoxide scavenger - Obstructive sleep apnoea syndrome $\bullet$ Upper airway muscles

\section{Corresponding author}

J. R. Skelly, C330 Health Sciences Centre, University College Dublin, Belfield, Dublin 4, Ireland. Tel: +353-86-3727847/0044 79801 55553. E-mail: jamesrichardskelly@gmail.com

\section{Introduction}

Skeletal muscles of the upper airway function to stabilize and dilate the pharyngeal airspace during inspiration. Impaired upper airway muscle function is implicated in obstructive sleep apnoea syndrome (OSAS) - a common and devastating respiratory disorder (White et al. 2005). Of interest, there is evidence of upper airway muscle remodelling and dysfunction in OSAS (Stauffer $e t$ al. 1989, Sériès et al. 1996b, Ferini-Strambi et al. 1998, Carrera et al. 1999) effects that resolve with continuous positive airway pressure (CPAP) treatment (Carrera et al. 1999). In recent years, translational animal models have emerged providing strong evidence that intermittent hypoxia - a hallmark feature of OSAS due to recurrent apnoea - is a key factor in the development of significant morbidities in OSAS patients. Several groups have demonstrated that intermittent hypoxia alters respiratory control (Ling et al. 2001, O'Halloran et al. 2002, Veasey et al. 2004), and causes respiratory muscle dysfunction (McGuire et al. 2002a, Pae et al. 2005) which may have special relevance to OSAS and other respiratory muscle weakness disorders. 
OSAS is now widely recognized as an oxidative stress disorder (Lavie et al. 2003, 2004, 2009) and antioxidant supplementation was recently shown to be effective in the treatment of OSAS patients (Sadasivam et al. 2011). There is substantial evidence to indicate that reactive oxygen species (ROS) are produced following bouts of hypoxia and reoxygenation in skeletal muscle. Though basal levels of ROS play an important role in a variety of physiological functions including optimum contractile function in muscle (Reid et al. 1993), ROS-induced skeletal muscle dysfunction is well described (Anzueto et al. 1994, Supinski 1998, Dunleavy et al. 2008) and may contribute to the pathophysiology of OSAS (Lavie 2009). The first aim of this study was to compare the effects of sustained hypoxia, intermittent hypoxia and, hypoxia followed by re-oxygenation on rat pharyngeal dilator muscle function. We hypothesized that sternohyoid muscle dysfunction would be greatest in intermittent hypoxia treated muscles due to increased oxidative stress. The sternohyoid muscle was selected for study as it is readily accessible and has fibres running in a uniform direction facilitating the interpretation of isometric force measurements in vitro. Supra- and infra-hyoid muscles contribute to the regulation of pharyngeal airway calibre and sternohyoid muscle dysfunction has been reported in animal models of sleep-disordered breathing (Petrof et al. 1994, McGuire et al. 2002b,c, O'Halloran et al. 2002, Bradford et al. 2005, Pae et al. 2005, Dunleavy et al. 2008, Skelly et al. 2010b).

Pharmacotherapy for OSAS is considered a viable clinical option (Hudgel and Thanakitcharu 1998, Veasey 2003, Hedner et al. 2008) especially for patients for whom CPAP - the gold standard treatment for OSAS - is not well tolerated. Drugs that improve pharyngeal dilator muscle performance are likely to benefit some patients and anti-oxidant agents are promising in this regard (Jordan et al. 2006, Lee et al. 2009, Skelly et al. 2010a, 2012, Sadasivam et al. 2011). Therefore, the second aim of this study was to examine the efficacy of Tempol, a membrane-permeant superoxide dismutase (SOD)-mimetic, in mitigating respiratory muscle dysfunction secondary to gas treatments. We hypothesized that Tempol would be more effective in rescuing force following hypoxia/reoxygenation protocols compared to sustained hypoxia because of its free radical scavenging properties.

\section{Methods}

\section{Animals}

Experiments were performed on 32 adult male Wistar rats (body mass 195-315 g). Animals were assigned to one of four groups: control $(\mathrm{n}=7)$, sustained hypoxia $(\mathrm{SH} ; \mathrm{n}=8)$, intermittent hypoxia $(\mathrm{IH} ; \mathrm{n}=9)$ or hypoxia/re-oxygenation (HR; $\mathrm{n}=8$ ). All protocols described in this study were approved and carried out in accordance with local institutional guidelines.

\section{In vitro muscle preparation}

The animals were anaesthetized with $5 \%$ isoflurane and euthanized by cervical spinal cord section. The paired sternohyoid muscles were separated along their medial border and excised. The muscles were placed in a tissue bath at room temperature containing continuously gassed $\left(95 \% \mathrm{O}_{2} / 5 \% \mathrm{CO}_{2}\right)$ Krebs solution. The solution contained the following components: $\mathrm{NaCl}$ $120 \mathrm{mM}, \mathrm{KCl} 5 \mathrm{mM}, \mathrm{Ca}^{2+}$ gluconate $2.5 \mathrm{mM}, \mathrm{MgSO}_{4}$ $1.2 \mathrm{mM}, \mathrm{NaH}_{2} \mathrm{PO}_{4} 1.2 \mathrm{mM}, \mathrm{NaHCO}_{3} 25 \mathrm{mM}$ and glucose $11.5 \mathrm{mM}$. Longitudinal strips of muscle $(\sim 1 \mathrm{~mm}$ diameter) were prepared and placed vertically in Plexiglas tissue holders in Krebs solution in waterjacketed organ baths at $35{ }^{\circ} \mathrm{C}$ gassed with a $95 \% \mathrm{O}_{2} / 5 \%$ $\mathrm{CO}_{2}$ gas mixture. The neuromuscular paralyzing agent, d-tubocurarine $(25 \mu \mathrm{M})$, was added to the bathing medium to eliminate activation of intramuscular nerve branches thus ensuring that isometric force generation was due solely to direct muscle stimulation. The muscle bundles were positioned between a pair of platinum plate electrodes, with the base fixed to an immobile hook and the other end tied to an isometric force transducer with non-elastic string. The position of the force transducer could be adjusted by a micropositioner thus altering the length of the muscle strips. A fixed graduated scale located behind the muscle strip allowed the accurate measurement of muscle length.

\section{Protocol}

The optimum length (i.e. muscle length producing maximal isometric twitch force in response to supra-maximal stimulation) was determined by adjusting muscle length between intermittent stimulations. Once determined the muscle remained at this length for the full protocol. Next, the muscle was allowed a $5 \mathrm{~min}$ equilibration period. Peak tetanic force was determined in response to a $300 \mathrm{~ms}$ train at $100 \mathrm{~Hz}$. Tissue baths were then emptied and re-filled with Krebs \pm antioxidant and 
the bundles were exposed to one of four gas treatments (see below). After $30 \mathrm{~min}$, contractile and endurance properties were determined in response to electrical field stimulation delivered via plate electrodes flanking the tissue connected to a square pulse constant current stimulator. Data were recorded using a commercial data acquisition system (PowerLab, AD Instruments) and stored for later analysis on a computer. First, forcefrequency relationship was determined by sequentially stimulating the muscle strips at 10, 20, 30, 40, 60, 80 and $100 \mathrm{~Hz}$ for $300 \mathrm{~ms}$ at each stimulus frequency allowing a 2 min recovery interval between each stimulus (Fig. 1A). Two min following the force-frequency protocol, repeated muscle contraction was induced by stimulation at $40 \mathrm{~Hz}$ with $300 \mathrm{~ms}$ trains every $2 \mathrm{~s}$ for a period of $2.5 \mathrm{~min}$.

\section{Gas and antioxidant treatments}

For control studies, muscle bundles remained in Krebs solution gassed with a hyperoxic gas mixture (95\% $\mathrm{O}_{2} / 5 \% \mathrm{CO}_{2}$ ). We have previously established that sternohyoid muscle bundles incubated at $35^{\circ} \mathrm{C}$ generate higher forces in hyperoxia compared to a normoxic $(21 \%$ $\mathrm{O}_{2} / 5 \% \mathrm{CO}_{2}$ ) bathing medium (Skelly et al. 2010a). For $\mathrm{SH}$, muscle bundles were exposed to an anoxic gas mixture $\left(95 \% \mathrm{~N}_{2} / 5 \% \mathrm{CO}_{2}\right.$; producing a tissue bath $\mathrm{PO}_{2}$ of $\sim 40 \quad \mathrm{~mm} \mathrm{Hg}$ ) and contractile and endurance performance was assessed in hypoxia. For HR, muscle bundles were exposed to an anoxic gas mixture (95\% $\mathrm{N}_{2} / 5 \% \mathrm{CO}_{2}$ ) for $15 \mathrm{~min}$ followed by a hyperoxic gas mixture $\left(95 \% \mathrm{O}_{2} / 5 \% \mathrm{CO}_{2}\right)$ for $15 \mathrm{~min}$. Finally, for $\mathrm{IH}$, bundles were exposed to $3 \times 5 \mathrm{~min}$ anoxic gas treatment ( $95 \% \mathrm{~N}_{2} / 5 \% \mathrm{CO}_{2}$ ) interspersed with 5 min intervals of hyperoxia $\left(\begin{array}{lllll}95 & \mathrm{O}_{2} / 5 & \% & \mathrm{CO}_{2}\end{array}\right)$. Muscle function was assessed under hyperoxic conditions in all groups with the exception of the SH group. Studies were performed in the absence (control) or presence of 4-hydroxy2,2,6,6-tetramethylpiperidine-1-oxyl (Tempol, $10 \mathrm{mM}$ ) performed in parallel using a double tissue bath set-up allowing paired comparisons for each of the four gas treatments. All compounds were purchased from SigmaAldrich Company, Dublin, Ireland and made up fresh each day.

\section{Data analysis}

Specific force was calculated in $\mathrm{N} / \mathrm{cm}^{2}$ of muscle cross-sectional area. The latter was approximated by weighing the dry muscle strips at the end of the experimental protocol and dividing this by the product of optimal length and muscle density (assumed to be $1.056 \mathrm{~g} / \mathrm{cm}^{3}$ ). The force transducers were calibrated using known gramme weights. Peak tetanic force (Fmax) was determined in response to $100 \mathrm{~Hz}$ stimulation at the beginning of the protocol. For the force-frequency relationship, data across the range of stimulus frequencies employed in the study were normalized to the initial peak force determined at the beginning of the study. Additionally, non-linear regression (curve-fit) analysis was employed (Graph Pad Prism) for all groups, allowing us to determine minimum, maximum, slope and $\mathrm{EF}_{50}$ values (i.e. stimulus frequency producing $50 \%$ of peak force) for sternohyoid muscle following each of the gas treatments \pm Tempol. Finally, to assess muscle performance in response to repeated activation we performed two sets of analyses. First, each tetanic contraction was measured and specific force was averaged in $10 \mathrm{~s}$ bins (i.e. 5 consecutive tetanic contractions) over the initial $1 \mathrm{~min}$ and in $30 \mathrm{~s}$ bins over the remaining $90 \mathrm{~s}$. Second, a performance index was calculated for the whole fatigue trial according to the following: [average twitch amplitude of all 60 contractions/initial twitch amplitude $x$ 100] modified from Healy et al. (2008). Values are expressed as mean \pm SEM. Statistical comparisons between groups were performed using one-way (gas treatment) or two-way ANOVA (drug x gas treatment) and Bonferroni post-hoc tests. $\mathrm{P}<0.05$ was the criterion for significance in all tests.

\section{Results}

\section{Effect of gas treatment on sternohyoid muscle force}

Prior to commencing gas treatments, peak tetanic force in response to $100 \mathrm{~Hz}$ stimulation (Fmax) was similar in all four groups $(\mathrm{P}=0.9705$, one-way ANOVA; Fig. 1B). The effect of gas treatment on rat upper airway muscle force is illustrated in Figure 1C. For each preparation, data are normalized to the initial peak force (Fmax) determined before gas treatments. Both IH and HR had a significant depressor effect on muscle force compared to control (Fig. 1C and 1D). The negative inotropic effect of the two gas treatments was equivalent (Fig. 1C and 1D). As expected, SH significantly depressed sternohyoid muscle force (Fig. 1C and 1D). 
A

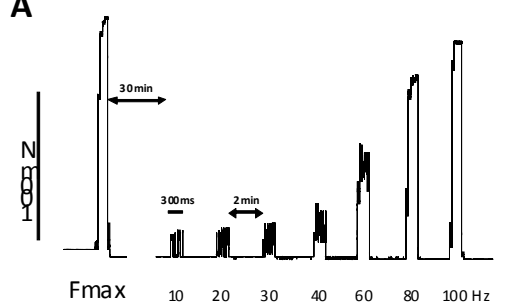

C

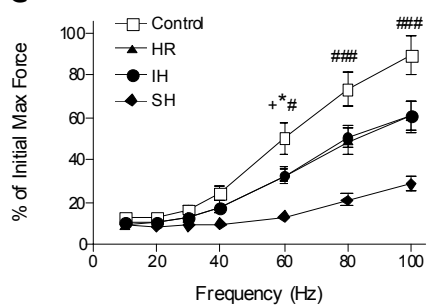

B

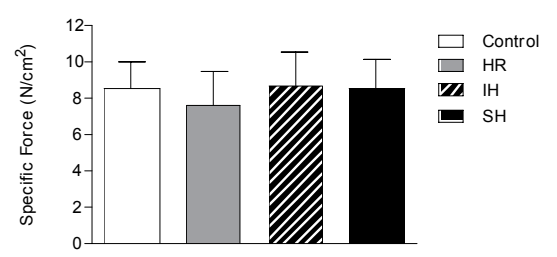

D

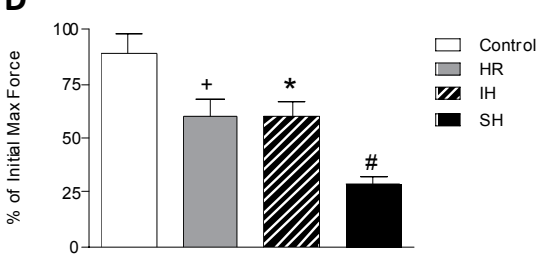

Fig. 1.(A) Original traces of peak isometric force (Fmax) and force-frequency protocol (incremental stimulus frequencies of $10-20 \mathrm{~Hz}$ up to $100 \mathrm{~Hz}$ ) in drug-free Krebs under control (hyperoxic) gas conditions. (B) Values (mean \pm SEM.) for Fmax in all four gas conditions. (C) Values for force-frequency relationship in all groups normalized to initial Fmax. Data show that hypoxia/re-oxygenation (HR), intermittent hypoxia (IH) and sustained hypoxia ( $\mathrm{SH}$ ) caused a significant decrease in isometric force compared to control muscles [HR, IH, and SH at100 Hz (\#), p<0.001; HR, $\mathrm{IH}$, and $\mathrm{SH}$ at $80 \mathrm{~Hz}(\#), \mathrm{p}<0.001 ; \mathrm{SH}$ at $60 \mathrm{~Hz}(\#), \mathrm{p}<0.001 ; \mathrm{IH}$ at $60 \mathrm{~Hz}(*), \mathrm{p}<0.01$; $\mathrm{HR}$ at $60 \mathrm{~Hz}(+), \mathrm{p}<0.05$; one-way ANOVA]. (D) Values (mean \pm SEM) for peak force in each gas group normalized to initial Fmax. Note the significantly lower force in $H R$, IH and $\mathrm{SH}$ compared to control muscles $(\mathrm{HR}(+)$, $\mathrm{p}<0.05 ; \mathrm{IH}(*), \mathrm{p}<0.01 ; \mathrm{SH}(\#), \mathrm{p}<0.001$; one-way ANOVA).

Fig. 2. Values (mean \pm SEM) for forcefrequency relationship normalized to Fmax in all four gas states \pm Tempol. Tempol increased force in Control $(P=0.0715), H R$ $(P=0.0068)$ IH $\quad(P<0.0001)$ and $\mathrm{SH}$ $(\mathrm{P}<0.0001) ; \quad$ two-way ANOVA. Post-hoc analysis revealed: $\mathrm{IH}$ at $60 \mathrm{~Hz}(*), \mathrm{p}<0.01$ and $80 \mathrm{~Hz}(+), \mathrm{p}<0.05$; $\mathrm{SH}$ at $60 \mathrm{~Hz}(+), \mathrm{p}<0.05$; $80 \mathrm{~Hz}(+), \mathrm{p}<0.05$ and $100 \mathrm{~Hz}(*), \mathrm{p}<0.01$.
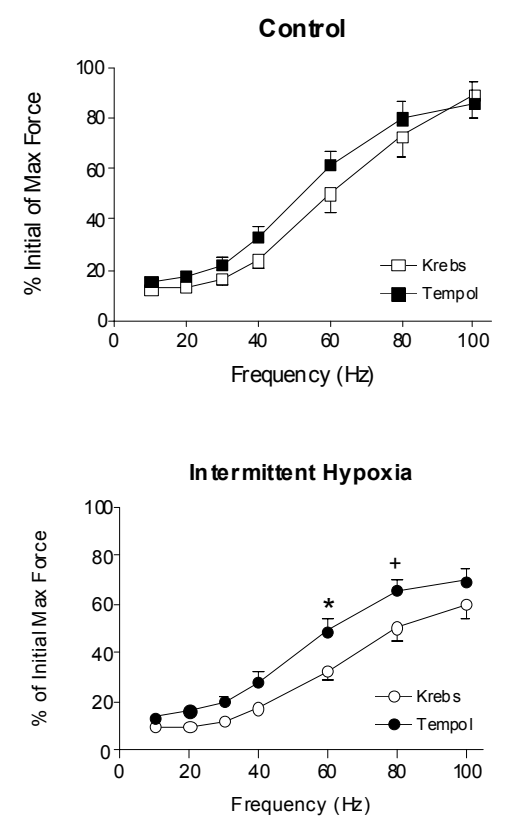

A

B

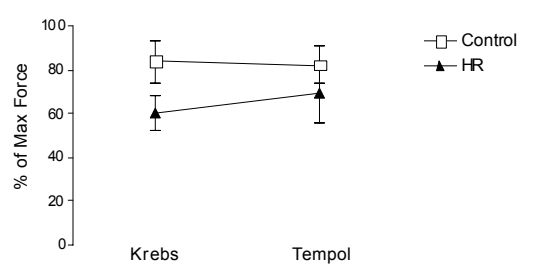

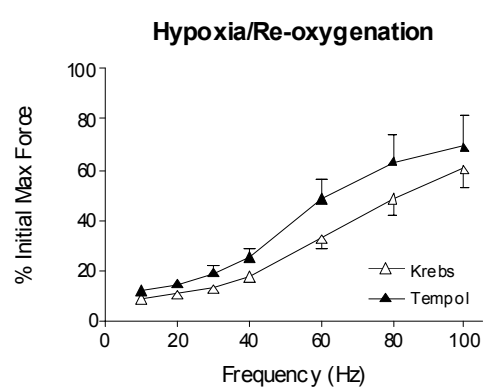

Sustained Hypoxia

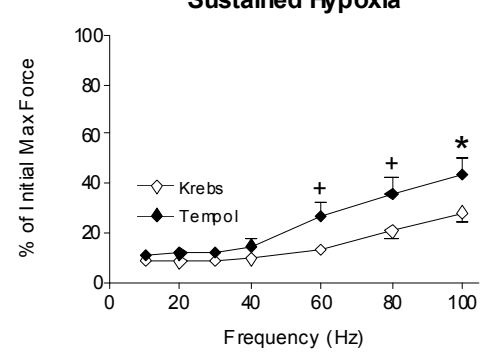

Fig. 3. Values (mean \pm SEM) for peak isometric force normalized to Fmax in muscle bundles incubated in Krebs \pm Tempol following hypoxia-reoxygenation (HR), intermittent hypoxia (IH), and sustained hypoxia (SH). (A) Two-way ANOVA revealed that there was no significant effect of gas treatment $(p=0.0711)$ or Tempol $(p=0.7172)$. The interaction term ( $\mathrm{HR} \times$ Tempol) was also not significant $(p=0.5901)$. (B) There was a significant effect of gas treatment $(p=0.0226)$ but not Tempol $(p=0.6195)$. The interaction term (IH $x$ Tempol) was not significant $(p=0.4666)$. (C) There was a significant effect of gas treatment $(p<0.0001)$ but not Tempol $(p=0.3640)$. The interaction term (SH $x$ Tempol) was not significant $(p=0.2555)$. 
Effect of Tempol on sternohyoid muscle force

Fmax was equivalent in all four groups prior to commencing gas + Tempol treatments $(\mathrm{P}=0.7722$, oneway ANOVA). Tempol partially ameliorated sternohyoid muscle force decline following IH, HR and SH causing a significant leftward shift in the force-frequency relationship (Fig. 2). However, two-way ANOVA comparing control and individual gas treatments indicated that there was no significant gas $\mathrm{x}$ Tempol interaction for force-frequency relationships. Peak force decline in IH, HR and $\mathrm{SH}$ groups was unaffected by Tempol treatment (Fig. 3). $\mathrm{EF}_{50}$ was significantly decreased by Tempol independent of gas treatment (Fig. 4).

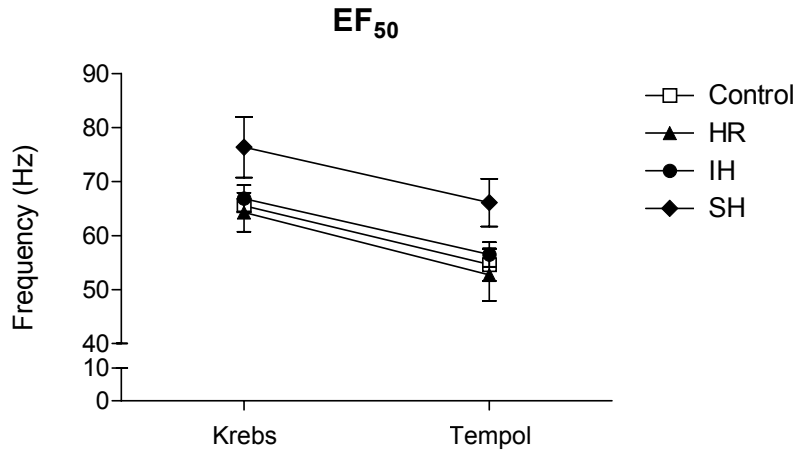

Fig. 4. Values for $\mathrm{EF}_{50}$ for all four gas conditions \pm Tempol. Tempol significantly decreased $\mathrm{EF}_{50}$ independent of gas treatment $(p=0.0002$, two-way ANOVA).
A

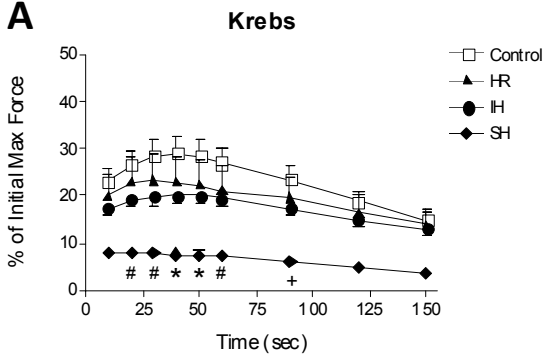

C

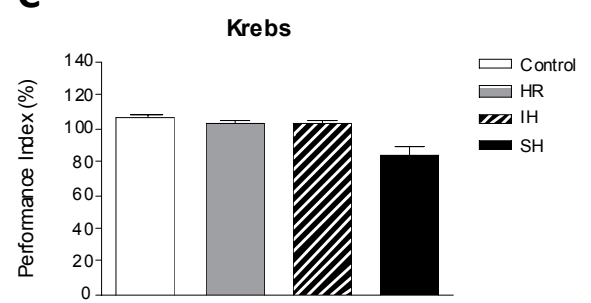

B

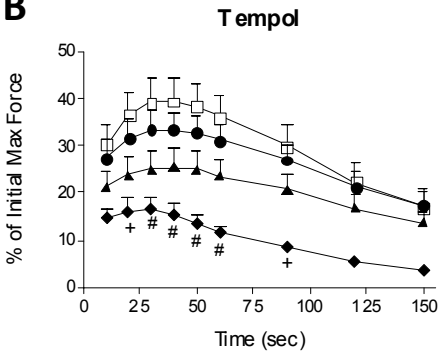

D

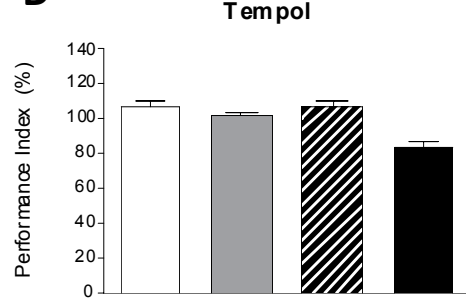

Fig. 5. Values (mean \pm SEM) for sternohyoid muscle force (normalized to Fmax) during repeated stimulation in all four gas conditions in Krebs (A) and Tempoltreated (B) muscle bundles. Gas treatments significantly decreased force during repeated muscle stimulation (HR: $\mathrm{P}=0.0326$; IH: $\mathrm{P}<0.0001 ; \mathrm{SH}: \mathrm{P}<0.0001$, two-way ANOVA). Post-hoc analysis for A: $\mathrm{SH}$ at $20 \mathrm{~s}(\#)$, $\mathrm{p}<0.01$; at $30 \mathrm{~s}(\#), \mathrm{p}<0.01$; at $40 \mathrm{~s}\left({ }^{*}\right)$, $\mathrm{p}<0.001$; at $50 \mathrm{~s}(*), \mathrm{p}<0.001 ;$ at $60 \mathrm{~s}(\#)$, $\mathrm{p}<0.01$, and at $90 \mathrm{~s}(+), \mathrm{p}<0.05$. Post-hoc analysis for $\mathrm{B}$ : $\mathrm{SH}$ at $20 \mathrm{~s}(+), \mathrm{p}<0.05$; at $30 \mathrm{~s}(\#), \mathrm{p}<0.01$; at $40 \mathrm{~s}(\#), \mathrm{p}<0.01$; at $50 \mathrm{~s}(\#), \mathrm{p}<0.01$; at $60 \mathrm{~s}(\#), \mathrm{p}<0.01$, and at $90 \mathrm{~s}(+), \mathrm{p}<0.05$. Performance index was significantly decreased by $\mathrm{SH}(p<0.0001$, two-way ANOVA) (C and D). There was no significant effect of Tempol and no significant interaction ( $\mathrm{SH} \times \mathrm{Tempol}$ ).
Sternohyoid muscle performance during repeated stimulation

Data illustrating sternohyoid muscle performance during repeated stimulation in each of the four gas treatments in the absence and presence of Tempol are shown in Figures 5A and 5B respectively. The performance index was significantly decreased by SH, but not by IH or HR treatments (Fig. 5C and 5D). Tempol failed to recover $\mathrm{SH}$-induced decrease in sternohyoid muscle performance (Fig. 5C and 5D).

\section{Discussion}

The main findings of the present study are: 1) IH and HR have an equivalent negative inotropic effect on isolated rat sternohyoid muscle force; 2) Tempol increases sternohyoid muscle sensitivity to electrical stimulation and was modestly effective in preventing muscle weakness following gas treatments; 3) $\mathrm{SH}$, but not IH or HR treatments, decreased sternohyoid muscle endurance properties. Tempol, however, failed to recover decreased upper airway muscle performance during $\mathrm{SH}$.

OSAS is now recognized as an oxidative stress disorder (Lavie et al. 2003, 2004, 2009) and upper airway muscle dysfunction in OSAS patients may be ROSdependent. Free radicals are vital for a number of physiological functions, including optimum muscle contraction (Reid et al. 1993). Excitation-contraction coupling in skeletal muscle involves alterations in the redox state of several key proteins central to muscle contraction. Cellular redox balance is dynamic, and when free radicals are produced in excess of a cell's capacity to scavenge them, oxidative stress ensues leading to cellular dysfunction. ROS-mediated skeletal muscle dysfunction 
is well described (Anzueto et al. 1994, Supinski 1998, Dunleavy et al. 2008). The mechanical performance of the pharyngeal dilator muscles is a critical determinant of pharyngeal airway stability and hence respiratory homeostasis. We observed that IH and HR both depressed sternohyoid muscle force. Our experimental design was such that we measured peak specific force in all groups prior to commencing experimental protocols, and we established that forces were not different when groups were compared. Therefore differences across groups can be attributed solely to gas treatment and are not likely related to group differences per se. Of interest, the two gas protocols decreased pharyngeal dilator muscle force to a similar extent. Cumulatively, both protocols exposed the sternohyoid muscle bundles to $15 \mathrm{~min}$ of hypoxia and 15 min of hyperoxia. That is, the duration and intensity of the stimuli were identical in the two protocols, but the pattern of exposure was (marginally) different. It would be interesting to assess if further variations in the pattern of IH enhanced the negative inotropic effect. The decrement in muscle force following IH and HR can be attributed to a number of mechanisms. Oxidative modification of regulatory proteins such as sarcolemmal potassium channels (Sen et al. 1995, Dudley et al. 2006), sarcoplasmic reticulum calcium release (RYR) and reuptake (SERCA) channels (Donoso et al. 2011), troponin (Putkey et al. 1993), tropomyosin (Williams and Swenson 1982), actin (Hinshaw et al. 1991) myosin light (Sweeney et al. 1993) and heavy chains (Brooke and Kaiser 1970, Ajtai and Burghardt 1989) could account for free radical-mediated decreases in skeletal muscle force.

Previous studies point to ROS-mediated modification of myofibrillary calcium sensitivity (Bruton et al. 2008), but in the present study $\mathrm{EF}_{50}$ (a measure of sensitivity to stimulation) was minimally affected by gas treatments suggesting that sensitivity to electrical stimulation was unchanged by gas treatment. Force decline was observed in response to stimulus frequencies ranging $60-100 \mathrm{~Hz}$, which one could argue is in the supra-physiological range as motor neuronal firing and muscle fibre recruitment occurs in vivo over $10-50 \mathrm{~Hz}$ in a typical skeletal muscle. However, instantaneous firing frequencies in excess of $100 \mathrm{~Hz}$ have been observed in human upper airway muscles (Farina and Falla 2009). Thus, we argue that the depressant effect of IH and HR is not only physiologically relevant but may be especially relevant to upper airway dilator muscles and OSAS, where recruitment of these muscles to recover airway collapse can occur hundreds of times over the course of the night in some patients (American Academy of Sleep Medicine Task Force 1999). One could speculate that impaired force-generating capacity in upper airway muscles following $\mathrm{IH}$ increases the vulnerability of the upper airway to collapse and/or impairs the ability to restore airway calibre following an occlusive event, given that recruitment of the pharyngeal dilator muscles is essential in this regard. We suggest that IH may contribute to upper airway muscle dysfunction in OSAS patients and may, at least in part, explain why the severity of occlusive events increases with time over the course of the night (Charbonneau et al. 1994).

As expected, sternohyoid muscle force was significantly depressed in SH. Hypoxia is a known depressor of force generation through actions on ion channel membrane conductance and reductions in sarcolemmal excitability leading to impaired excitationcontraction coupling. Also, under hypoxic conditions, several changes in the internal milieu such as acidosis and alterations in the concentration of inorganic phosphate and adenine nucleotides are capable of directly depressing the function of the contractile apparatus (Godt and Nosek 1989). The SH group was included as a reference benchmark of maximum hypoxic depression in muscle. However, comparison of the magnitude of force decline observed in SH with that seen in the IH and HR groups should be made with caution as contractile and endurance parameters were assessed in hyperoxia (control conditions) in the latter groups. Therefore, the negative inotropic effect of SH may be more representative of maximal force depression. It is plausible that hypoxic preconditioning during the $30 \mathrm{~min}$ gas exposure period may have altered the magnitude of hypoxic depression in sternohyoid muscle determined during the muscle function study. Indeed, muscle bundles incubated in hyperoxic conditions and then switched to hypoxia show $\sim 30 \%$ reduction in peak isometric force compared to the $\sim 50 \%$ reduction in peak force shown in Figure 3. Thus, as expected, longer durations of hypoxia are associated with decreased muscle function. Of note, however, $\mathrm{EF}_{50}$ values and performance index are equivalent in the above two hypoxic groups.

ROS are implicated in skeletal muscle fatigue and increased fatigue is reported in upper airway muscles from OSAS patients (Sériès et al. 1996a). Thus, we hypothesized that hypoxia/re-oxygenation protocols would compromise pharyngeal dilator muscle endurance. However, sternohyoid muscle performance index, which assesses all components of the fatigue trial (potentiation 
and run down) was similar in control and treatment groups, though specific forces were lower in treatment groups compared to control. It is conceivable that chronic exposure to HR cycles is necessary for muscle remodelling leading to the development of a more fatigable phenotype such as that seen in OSAS and rat models of chronic IH (McGuire et al. 2002b). Sternohyoid muscle performance index was significantly decreased during $\mathrm{SH}$ exposure.

Pharmacological therapy has been suggested as a clinical strategy in the treatment of OSAS (Hudgel and Thanakitcharu 1998, Veasey 2003, Hedner et al. 2008) and agents that improve upper airway muscle performance could serve as viable adjunct therapies in patients with sleep-disordered breathing. Previous work has characterized the action of a number of pharmacological agents on isolated upper airway muscle function with some encouraging results (Van Lunteren et al. 1995, 1996, Cantillon and Bradford 2001, O'Halloran 2006, Skelly et al. 2010a). Since reactive oxygen species (ROS) are implicated in skeletal muscle function and antioxidants have been shown to improve skeletal muscle performance (Reid et al. 1992, Reid 2008, Skelly et al. 2010a) including diaphragm (Wright et al. 2005) and pharyngeal dilator muscle function (Skelly et al. 2010a) we sought to test the efficacy of an antioxidant on sternohyoid muscle function in acute models manipulating tissue oxygenation. Tempol increased muscle sensitivity to stimulation (i.e. caused a decrease in the $\mathrm{EF}_{50}$ ) in all groups, but only modestly ameliorated force decline in the $\mathrm{IH}$ and $\mathrm{SH}$ treatment groups, and Tempol had no effect on sternohyoid muscle peak isometric force. Our findings are incongruent with observations that superoxide scavengers protect diaphragm (Mohanraj et al. 1998, Wright et al. 2005) and upper airway (Skelly et al. 2010a) muscle contractile function in severe hypoxia. Our findings do not support our original hypothesis that Tempol would prove more effective in preventing muscle dysfunction in IH and HR treated muscles due to its free radical scavenging properties. In addition, Tempol was ineffective in preventing decreased sternohyoid muscle performance during $\mathrm{SH}$, suggesting that muscle fatigue in hypoxia is not primarily driven by oxidative stress.

It is important to recognize, however, that we may not have employed the optimum concentration of Tempol in our study. We chose one concentration of Tempol in this study based on our previous report (Skelly et al. 2010a). It has been suggested that at high concentrations Tempol loses its protective action most likely because of an increased pro-oxidative action when superoxide levels are low (Edwards et al. 2007). Also, since ROS are required for optimal muscle function excessive scavenging of ROS could adversely affect muscle performance. The in vitro preparation described in this study could prove useful in the screening of established and novel antioxidant compounds with potential application in the treatment of respiratory muscle disorders. However, it should be noted that a plausible, if unexpected, conclusion of our study is that muscle weakness following IH and HR may relate, for the most part, to factors unrelated to oxidative stress.

In summary, we examined the effects of acute exposures to $\mathrm{IH}, \mathrm{HR}$ and $\mathrm{SH} \pm$ Tempol (a SOD-mimetic) on pharyngeal dilator muscle contractile and endurance properties in male rats. IH and HR both depressed sternohyoid muscle force but not fatigue compared to control. Tempol increased sensitivity to stimulation but failed to rescue pharyngeal dilator muscle force during gas challenges. Our in vitro model could prove useful in the screening of the effects of novel antioxidant compounds on upper airway muscle performance.

\section{Conflict of Interest}

There is no conflict of interest.

\section{Acknowledgements}

Funded by the Health Research Board Ireland (RP/2006/140). JRS is a UCD Ad Astra Research Scholar.

\section{References}

AJTAI K, BURGHARDT TP: Fluorescent modification and orientation of myosin sulfhydryl 2 in skeletal muscle fibers. Biochemistry 28: 2204-2210, 1989.

AMERICAN ACADEMY OF SLEEP MEDICINE TASK FORCE: Sleep-related breathing disorders in adults: recommendations for syndrome definition and measurement techniques in clinical research. Sleep 22: 667-689, 1999. 
ANZUETO A, SUPINSKI GS, LEVINE SM, JENKINSON SG: Mechanisms of disease: are oxygen-derived free radicals involved in diaphragmatic dysfunction? Am J Respir Crit Care Med 149: 1048-1052, 1994.

BRADFORD A, MCGUIRE M, O'HALLORAN KD: Does episodic hypoxia affect upper airway dilator muscle function? Implications for the pathophysiology of obstructive sleep apnoea. Respir Physiol Neurobiol 147: 223-234, 2005.

BROOKE MH, KAISER KK: Three "myosin adenosine triphosphatase" systems: the nature of their pH lability and sulfhydryl dependence. J Histochem Cytochem 18: 670-672, 1970.

BRUTON JD, PLACE N, YAMADA T, SILVA JP, ANDRADE FH, DAHLSTEDT AJ, ZHANG SJ, KATZ A, LARSSON NG, WESTERBLAD H: Reactive oxygen species and fatigue-induced prolonged low-frequency force depression in skeletal muscle fibres of rats, mice and SOD2 overexpressing mice. J Physiol 586: 175184, 2008.

CANTILLON D, BRADFORD A: Effect of almitrine on upper airway muscle contraction in young and old rats. Eur $J$ Pharmacol 412: 187-194, 2001.

CARRERA M, BARBE F, SAULEDA J, TOMAS M, GOMEZ C, AGUSTI AG: Patients with obstructive sleep apnea exhibit genioglossus dysfunction that is normalized after treatment with continuous positive airway pressure. Am J Respir Crit Care Med 159: 1960-1966, 1999.

CHARBONNEAU M, MARIN JM, OLHA A, KIMOFF RJ, LEVY RD, COSIO MG: Changes in obstructive sleep apnea characteristics through the night. Chest 106: 1695-1701, 1994.

DONOSO P, SANCHEZ G, BULL R, HIDALGO C: Modulation of cardiac ryanodine receptor activity by ROS and RNS. Front Biosci 16: 553-567, 2011.

DUDLEY RW, DANIALOU G, GOVINDARAJU K, LANDS L, EIDELMAN DE, PETROF BJ: Sarcolemmal damage in dystrophin deficiency is modulated by synergistic interactions between mechanical and oxidative/nitrosative stresses. Am J Pathol 168: 1276-1287; 1404-1275, 2006.

DUNLEAVY M, BRADFORD A, O'HALLORAN KD: Oxidative stress impairs upper airway muscle endurance in an animal model of sleep-disordered breathing. Adv Exp Med Biol 605: 458-462, 2008.

EDWARDS JN, MACDONALD WA, VAN DER POEL C, STEPHENSON DG: O2(*-) production at 37 degrees C plays a critical role in depressing tetanic force of isolated rat and mouse skeletal muscle. Am J Physiol Cell Physiol 293: C650-C660, 2007.

FARINA D, FALLA D: Discharge rate of sternohyoid motor units activated with surface EMG feedback. J Neurophysiol 101: 624-632, 2009.

FERINI-STRAMBI LJ, SMIRNE S, MOZ U, SFERRAZZA B, IANNACCONE S: Muscle fibre type and obstructive sleep apnea. Sleep Res Online 1: 24-27, 1998.

GODT RE, NOSEK TM: Changes of intracellular milieu with fatigue or hypoxia depress contraction of skinned rabbit skeletal and cardiac muscle. J Physiol 412: 155-180, 1989.

HEALY CF, MCMORROW C, O'HERLIHY C, O'CONNELL PR, JONES JF: External anal sphincter fatigue is not improved by N-acetylcysteine in an animal model. Neurogastroenterol Motil 20: 719-724, 2008.

HEDNER J, GROTE L, ZOU D: Pharmacological treatment of sleep apnea: current situation and future strategies. Sleep Med Rev 12: 33-47, 2008.

HINSHAW DB, BURGER JM, BEALS TF, ARMSTRONG BC, HYSLOP PA: Actin polymerization in cellular oxidant injury. Arch Biochem Biophys 288: 311-316, 1991.

HUDGEL DW, THANAKITCHARU S: Pharmacologic treatment of sleep-disordered breathing. Am J Respir Crit Care Med 158: 691-699, 1998.

JORDAN W, COHRS S, DEGNER D, MEIER A, RODENBECK A, MAYER G, PILZ J, RUTHER E, KORNHUBER J, BLEICH S: Evaluation of oxidative stress measurements in obstructive sleep apnea syndrome. $J$ Neural Transm 113: 239-254, 2006.

LAVIE L: Obstructive sleep apnoea syndrome - an oxidative stress disorder. Sleep Med Rev 7: 35-51, 2003.

LAVIE L: Oxidative stress - a unifying paradigm in obstructive sleep apnea and comorbidities. Prog Cardiovasc Dis 51: 303-312, 2009.

LAVIE L, VISHNEVSKY A, LAVIE P: Evidence for lipid peroxidation in obstructive sleep apnea. Sleep 27: 123-128, 2004. 
LEE DS, BADR MS, MATEIKA JH: Progressive augmentation and ventilatory long-term facilitation are enhanced in sleep apnoea patients and are mitigated by antioxidant administration. J Physiol 587: 5451-5467, 2009.

LING L, FULLER DD, BACH KB, KINKEAD R, OLSON EB JR, MITCHELL GS: Chronic intermittent hypoxia elicits serotonin-dependent plasticity in the central neural control of breathing. J Neurosci 21: 5381-5388, 2001.

MCGUIRE M, CANTILLON D, BRADFORD A: Effects of almitrine on diaphragm contractile properties in young and old rats. Respiration 69: 75-80, 2002a.

MCGUIRE M, MACDERMOTT M, BRADFORD A: The effects of chronic episodic hypercapnic hypoxia on rat upper airway muscle contractile properties and fiber-type distribution. Chest 122: 1400-1406, $2002 \mathrm{~b}$.

MCGUIRE M, MACDERMOTT M, BRADFORD A: Effects of chronic episodic hypoxia on rat upper airway muscle contractile properties and fiber-type distribution. Chest 122: 1012-1017, 2002c.

MOHANRAJ P, MEROLA AJ, WRIGHT VP, CLANTON TL: Antioxidants protect rat diaphragmatic muscle function under hypoxic conditions. J Appl Physiol 84: 1960-1966, 1998.

O'HALLORAN KD: Effects of nicotine on rat sternohyoid muscle contractile properties. Respir Physiol Neurobiol 150: 200-210, 2006.

O'HALLORAN KD, MCGUIRE M, O'HARE T, BRADFORD A: Chronic intermittent asphyxia impairs rat upper airway muscle responses to acute hypoxia and asphyxia. Chest 122: 269-275, 2002.

PAE EK, WU J, NGUYEN D, MONTI R, HARPER RM: Geniohyoid muscle properties and myosin heavy chain composition are altered after short-term intermittent hypoxic exposure. J Appl Physiol 98: 889-894, 2005.

PETROF BJ, PACK AI, KELLY AM, EBY J, HENDRICKS JC: Pharyngeal myopathy of loaded upper airway in dogs with sleep apnea. J Appl Physiol 76: 1746-1752, 1994.

PUTKEY JA, DOTSON DG, MOUAWAD P: Formation of inter- and intramolecular disulfide bonds can activate cardiac troponin C. J Biol Chem 268: 6827-6830, 1993.

REID MB: Free radicals and muscle fatigue: Of ROS, canaries, and the IOC. Free Radic Biol Med 44: 169-179, 2008.

REID MB, HAACK KE, FRANCHEK KM, VALBERG PA, KOBZIK L, WEST MS: Reactive oxygen in skeletal muscle. I. Intracellular oxidant kinetics and fatigue in vitro. J Appl Physiol 73: 1797-1804, 1992.

REID MB, KHAWLI FA, MOODY MR: Reactive oxygen in skeletal muscle. III. Contractility of unfatigued muscle. J Appl Physiol 75: 1081-1087, 1993.

SADASIVAM K, PATIAL K, VIJAYAN VK, RAVI K: Anti-oxidant treatment in obstructive sleep apnoea syndrome. Indian J Chest Dis Allied Sci 53: 153-162, 2011.

SEN CK, KOLOSOVA I, HANNINEN O, ORLOV SN: Inward potassium transport systems in skeletal muscle derived cells are highly sensitive to oxidant exposure. Free Radic Biol Med 18: 795-800, 1995.

SÉRIÈS F, CÔTÉ C, SIMONEAU JA, ST PIERRE S, MARC I: Upper airway collapsibility, and contractile and metabolic characteristics of musculus uvulae. Faseb J 10: 897-904, 1996a.

SÉRIÈS F, SIMONEAU JA, ST PIERRE S, MARC I: Characteristics of the genioglossus and musculus uvulae in sleep apnea hypopnea syndrome and in snorers. Am J Respir Crit Care Med 153: 1870-1874, 1996 b.

SKELLY JR, BRADFORD A, JONES JF, O'HALLORAN KD: Superoxide scavengers improve rat pharyngeal dilator muscle performance. Am J Respir Cell Mol Biol 42: 725-731, 2010a.

SKELLY JR, BRADFORD A, O'HALLORAN KD: Intermittent hypoxia impairs pharyngeal dilator muscle function in male but not female rats. Adv Exp Med Biol 669: 285-287, $2010 \mathrm{~b}$.

SKELLY JR, EDGE D, SHORTT CM, JONES JF, BRADFORD A, O'HALLORAN KD: Tempol ameliorates pharyngeal dilator muscle dysfunction in a rodent model of chronic intermittent hypoxia. Am J Respir Cell Mol Biol 46: 139-148, 2012.

STAUFFER JL, BUICK MK, BIXLER EO, SHARKEY FE, ABT AB, MANDERS EK, KALES A, CADIEUX RJ, BARRY JD, W ZC: Morphology of the uvula in obstructive sleep apnea. Am Rev Respir Dis 140: 724-728, 1989.

SUPINSKI G: Free radical induced respiratory muscle dysfunction. Mol Cell Biochem 179: 99-110, 1998.

SWEENEY HL, BOWMAN BF, STULL JT: Myosin light chain phosphorylation in vertebrate striated muscle: regulation and function. Am J Physiol 264: C1085-C1095, 1993. 
VAN LUNTEREN E, VAFAIE H, MOYER M: Changes in pharyngeal respiratory muscle force produced by $\mathrm{K}^{+}$ channel blockade. Respir Physiol 99: 331-340, 1995.

VAN LUNTEREN E, VAFAIE H, MILLER MJ: Effects of theophylline on pharyngeal dilator and diaphragm muscle contractile properties. Respiration 63: 88-93, 1996.

VEASEY SC: Serotonin agonists and antagonists in obstructive sleep apnea: therapeutic potential. Am J Respir Med 2: 21-29, 2003.

VEASEY SC, ZHAN G, FENIK P, PRATICO D: Long-term intermittent hypoxia: reduced excitatory hypoglossal nerve output. Am J Respir Crit Care Med 170: 665-672, 2004.

WILLIAMS DL JR, SWENSON CA: Disulfide bridges in tropomyosin. Effect on ATPase activity of actomyosin. Eur J Biochem 127: 495-499, 1982.

WRIGHT VP, KLAWITTER PF, ISCRU DF, MEROLA AJ, CLANTON TL: Superoxide scavengers augment contractile but not energetic responses to hypoxia in rat diaphragm. J Appl Physiol 98: 1753-1760, 2005. 